\title{
A MODIFIED HALPERN-TYPE ITERATION PROCESS FOR AN EQUILIBRIUM PROBLEM AND A FAMILY OF RELATIVELY QUASI-NONEXPANSIVE MAPPINGS IN BANACH SPACES
}

\author{
PRASIT CHOLAMJIAK ${ }^{1}$ AND SUTHEP SUANTAI ${ }^{2 *}$ \\ Communicated by Yeol Je Cho
}

\begin{abstract}
In this paper, based on a generalized projection, we introduce a new modified Halpern-type iteration algorithm for finding a common element of the set of solutions of an equilibrium problem and the set of a common fixed point of an infinitely countable family of relatively quasi-nonexpansive mappings in the framework of Banach spaces. We establish the strong convergence theorem and obtain some applications. Our main results improve and extend the corresponding results announced by many authors.
\end{abstract}

\section{INTRODUCTION}

Let $H$ be a real Hilbert space and $C$ be a nonempty closed convex subset of $H$. Let $T: C \rightarrow C$ be a mapping. Recall that $T$ is said to be nonexpansive if $\|T x-T x\| \leq\|x-y\|$ for all $x, y \in H$. Denote by $F(T)$ the set of fixed points of $T$.

In 1967, Halpern [10] proposed a classical iterative process to approximate a fixed point of a nonexpansive mapping $T$ in a Hilbert space $H$. The following algorithm is known as Halpern's iteration process:

$$
x_{n+1}=\alpha_{n} u+\left(1-\alpha_{n}\right) T x_{n}, \quad \forall n \geq 1,
$$

Date: Received: 3 May 2010.

* Corresponding author

(c) 2010 N.A.G.

2000 Mathematics Subject Classification. 47H09, 47H10.

Key words and phrases. Equilibrium problem; strong convergence; common fixed point; relatively quasi-nonexpansive mapping; Halpern-type iteration process. 
where $u \in C$ is a fixed point and $\left\{\alpha_{n}\right\}$ is a sequence in $[0,1]$. Halpern [10] proved that the following conditions:

$$
\text { (i) : } \lim _{n \rightarrow \infty} \alpha_{n}=0, \quad \text { (ii) : } \sum_{n=1}^{\infty} \alpha_{n}=\infty
$$

are indeed necessary for the strong convergence of the algorithm (1.1) for all closed convex subsets $C$ of a Hilbert space $H$ and for all nonexpansive mappings $T$ on $C$; see also [29].

However, due to the restriction of condition (ii), the convergence of $\left\{x_{n}\right\}$ is believed to be slow. So to improve the rate of convergence of algorithm (1.1), one has to perform some additional step of iteration.

Question 1. Can we construct algorithms for modifying the iterative process (1.1) to have strong convergence under the condition (i) only ?

Recently, Martinez-Yanes and Xu [13] has adapted Nakajo and Takahashi's [16] idea to modify the process (1.1) for a nonexpansive mapping $T$ in a Hilbert space $H$ :

$$
\left\{\begin{array}{l}
x_{0} \in C \quad \text { chosen arbitrarily, } \\
y_{n}=\alpha_{n} x_{0}+\left(1-\alpha_{n}\right) T x_{n} \\
C_{n}=\left\{z \in C:\left\|y_{n}-z\right\|^{2} \leq\left\|x_{n}-z\right\|^{2}+\alpha_{n}\left(\left\|x_{0}\right\|^{2}+2\left\langle x_{n}-x_{0}, z\right\rangle\right)\right\} \\
Q_{n}=\left\{z \in C:\left\langle x_{n}-z, x_{n}-x_{0}\right\rangle \leq 0\right\} \\
x_{n+1}=P_{C_{n} \cap Q_{n}} x_{0} \quad n \geq 1
\end{array}\right.
$$

where $P_{K}$ is the metric projection from $H$ onto a closed convex subset $K$ of $H$. They proved that the sequence $\left\{x_{n}\right\}$ generated by above iterative scheme converges strongly to $P_{F(T)} x_{0}$ provided the sequence $\left\{\alpha_{n}\right\} \subset(0,1)$ satisfies $\lim _{n \rightarrow \infty} \alpha_{n}=$ 0 .

Question 2. Can we extend the iterative process (1.2) from Hilbert spaces to Banach spaces?

Very recently, Qin et al. [19], introduced the following modification of the process (1.2) for a closed relatively quasi-nonexpansive mapping $T$ in a Banach space $E$ :

$$
\left\{\begin{array}{l}
x_{0} \in E \quad \text { chosen arbitrarily, } \\
C_{1}=C, \quad x_{1}=\Pi_{C_{1}} x_{0} \\
y_{n}=J^{-1}\left(\alpha_{n} J x_{1}+\left(1-\alpha_{n}\right) J T x_{n}\right) \\
C_{n+1}=\left\{z \in C_{n}: \phi\left(z, y_{n}\right) \leq \alpha_{n} \phi\left(z, x_{1}\right)+\left(1-\alpha_{n}\right) \phi\left(z, x_{n}\right)\right\} \\
x_{n+1}=\Pi_{C_{n+1}} x_{1}, \quad \forall n \geq 1
\end{array}\right.
$$

where $J$ is the duality mapping on $E$ and $\Pi_{K}$ is the generalized projection from $E$ onto $K$. They proved that the sequence $\left\{x_{n}\right\}$ converges strongly to $\Pi_{F(T)} x_{1}$ provided $\lim _{n \rightarrow \infty} \alpha_{n}=0$.

Question 3. Can we extend the above algorithm to finding a common element of the set of solutions of an equilibrium problem and the set of a common fixed 
point of an infinitely countable family of relatively quasi-nonexpansive mappings in the framework of Banach spaces?

Let $f$ be a bifunction from $C \times C$ to the set of real numbers $\mathbb{R}$. The equilibrium problem is to find $\hat{x} \in C$ such that

$$
f(\hat{x}, y) \geq 0, \forall y \in C .
$$

The set of solutions of (1.4) is denoted by $E P(f)$.

For solving the equilibrium problem, let us assume that a bifunction $f$ satisfies the following conditions:

(A1) $f(x, x)=0$ for all $x \in C$;

(A2) $f$ is monotone, i.e. $f(x, y)+f(y, x) \leq 0$ for all $x, y \in C$;

(A3) for all $x, y, z \in C$, $\lim \sup _{t \downarrow 0} f(t z+(1-t) x, y) \leq f(x, y)$;

(A4) for all $x \in C, f(x,$.$) is convex and lower semicontinuous.$

The problem of finding a common element of the set of fixed points of nonexpansive, relatively nonexpansive or relatively quasi-nonexpansive mappings and the set of solutions of an equilibrium problem in the framework of Hilbert spaces and Banach spaces has been intensively studied by many authors; for instance, see [7, 9, 14, 15, 17, 22, 23, 25, 27, 28, 30] and the references cited therein.

Motivated and inspired by Martinez-Yanes and Xu [13, and Qin et al. [19], we construct a new hybrid projection algorithm for finding a common element of the set of solutions of an equilibrium problem and the set of a common fixed point of a family of closed relatively quasi-nonexpansive mappings in the framework of Banach spaces.

\section{Preliminaries AND lemmas}

Let $E$ be a real Banach space and let $U=\{x \in E:\|x\|=1\}$ be the unit sphere of $E$. A Banach space $E$ is said to be strictly convex if for any $x, y \in U$,

$$
x \neq y \text { implies }\left\|\frac{x+y}{2}\right\|<1 .
$$

It is also said to be uniformly convex if for each $\varepsilon \in(0,2]$, there exists $\delta>0$ such that for any $x, y \in U$,

$$
\|x-y\| \geq \varepsilon \text { implies }\left\|\frac{x+y}{2}\right\|<1-\delta .
$$

It is known that a uniformly convex Banach space is reflexive and strictly convex. Define a function $\delta:[0,2] \rightarrow[0,1]$ called the modulus of convexity of $E$ as follows:

$$
\delta(\varepsilon)=\inf \left\{1-\left\|\frac{x+y}{2}\right\|: x, y \in E,\|x\|=\|y\|=1,\|x-y\| \geq \varepsilon\right\} .
$$

Then $E$ is uniformly convex if and only if $\delta(\varepsilon)>0$ for all $\varepsilon \in(0,2]$. A Banach space $E$ is said to be smooth if the limit

$$
\lim _{t \rightarrow 0} \frac{\|x+t y\|-\|x\|}{t}
$$


exists for all $x, y \in U$. It is also said to be uniformly smooth if the limit (2.1) is attained uniformly for $x, y \in U$. The normalized duality mapping $J: E \rightarrow 2^{E^{*}}$ is defined by

$$
J(x)=\left\{x^{*} \in E^{*}:\left\langle x, x^{*}\right\rangle=\|x\|^{2}=\left\|x^{*}\right\|^{2}\right\}
$$

for all $x \in E$. If $E$ is a Hilbert space, then $J=I$, where $I$ is the identity mapping. It is also known that if $E$ is uniformly smooth, then $J$ is uniformly norm-to-norm continuous on each bounded subset of $E$; see 24 for more details.

Let $E$ be a smooth Banach space. The function $\phi: E \times E \rightarrow \mathbb{R}$ is defined by

$$
\phi(x, y)=\|x\|^{2}-2\langle x, J y\rangle+\|y\|^{2}
$$

for all $x, y \in E$. In a Hilbert space $H$, we have $\phi(x, y)=\|x-y\|^{2}$ for all $x, y \in H$.

Let $C$ be a closed convex subset of $E$, and let $T$ be mapping from $C$ into itself. A point $p$ in $C$ is said to be an asymptotic fixed point of $T$ [21] if $C$ contains a sequence $\left\{x_{n}\right\}$ which converges weakly to $p$ such that $\lim _{n \rightarrow \infty}\left\|x_{n}-T x_{n}\right\|=0$. The set of asymptotic fixed point of $T$ will be denoted by $\hat{F}(T)$. A mapping $T$ is said to be relatively nonexpansive [3, 4, 14] if $\hat{F}(T)=F(T)$ and $\phi(p, T x) \leq$ $\phi(p, x)$ for all $p \in F(T)$ and $x \in C$. The asymptotic behavior of a relatively nonexpansive mapping was studied in [3, 4]. $T$ is said to be $\phi$-nonexpansive, if $\phi(T x, T y) \leq \phi(x, y)$ for $x, y \in C$. $T$ is said to be relatively quasi-nonexpansive or quasi- $\phi$-nonexpansive if $F(T) \neq \emptyset$ and $\phi(p, T x) \leq \phi(p, x)$ for all $p \in F(T)$ and $x \in C$. It is obvious that the class of relatively quasi-nonexpansive mappings is more general than the class of relatively nonexpansive mappings [3, 4, 6, 14]. Recall that $T$ is closed if

$$
x_{n} \rightarrow x, T x_{n} \rightarrow y \quad \text { imply } \quad T x=y .
$$

We give some examples which are closed relatively quasi-nonexpansive; see [18].

Example 2.1. Let $E$ be a uniformly smooth and strictly convex Banach space and $A \subset E \times E^{*}$ be a maximal monotone mapping such that its zero set $A^{-1} 0 \neq \emptyset$. Then, $J_{r}=(J+r A)^{-1} J$ is a closed relatively quasi-nonexpansive mapping from $E$ onto $D(A)$ and $F\left(J_{r}\right)=A^{-1} 0$.

Example 2.2. Let $\Pi_{C}$ be the generalized projection from a smooth, strictly convex, and reflexive Banach space $E$ onto a nonempty closed convex subset $C$ of $E$. Then, $\Pi_{C}$ is a closed relatively quasi-nonexpansive mapping with $F\left(\Pi_{C}\right)=C$.

Lemma 2.3 (Kamimura and Takahashi [1]]). Let $E$ be a uniformly convex and smooth Banach space and let $\left\{x_{n}\right\},\left\{y_{n}\right\}$ be two sequences of $E$. If $\phi\left(x_{n}, y_{n}\right) \rightarrow 0$ and either $\left\{x_{n}\right\}$ or $\left\{y_{n}\right\}$ is bounded, then $\left\|x_{n}-y_{n}\right\| \rightarrow 0$ as $n \rightarrow \infty$.

Let $E$ be a reflexive, strictly convex and smooth Banach space and let $C$ be a nonempty closed and convex subset of $E$. The generalized projection mapping, 
introduced by Alber [1], is a mapping $\Pi_{C}: E \rightarrow C$, that assigns to an arbitrary point $x \in E$ the minimum point of the function $\phi(y, x)$, that is, $\Pi_{C} x=\bar{x}$, where $\bar{x}$ is the solution to the minimization problem

$$
\phi(\bar{x}, x)=\min \{\phi(y, x): y \in C\} .
$$

The existence and uniqueness of the operator $\Pi_{C}$ follows from the properties of the functional $\phi$ and strict monotonicity of the duality mapping $J$; for instance, see $[1,2,8,11,24]$. In a Hilbert space, $\Pi_{C}$ is coincident with the metric projection.

Lemma 2.4 (Alber [1] and Kamimura and Takahashi [11]). Let $C$ be a nonempty closed convex subset of a smooth, strictly convex, and reflexive Banach space E, let $x \in E$ and let $z \in C$. Then $z=\Pi_{C} x$ if and only if

$$
\langle y-z, J x-J z\rangle \leq 0, \quad \forall y \in C .
$$

Lemma 2.5 (Alber [1] and Kamimura and Takahashi [11]). Let $C$ be a nonempty closed convex subset of a smooth, strictly convex and reflexive Banach space E. Then

$$
\phi\left(x, \Pi_{C} y\right)+\phi\left(\Pi_{C} y, y\right) \leq \phi(x, y) \quad \forall x \in C \text { and } y \in E .
$$

Lemma 2.6 (Qin et al. [18]). Let $E$ be a uniformly convex, smooth Banach space, let $C$ be a closed convex subset of $E$, let $T$ be a closed and relatively quasinonexpansive mapping from $C$ into itself. Then $F(T)$ is a closed convex subset of $C$.

Lemma 2.7 (Blum and Oettli [5]). Let $C$ be a closed convex subset of a smooth, strictly convex, and reflexive Banach space $E$, let $f$ be a bifunction from $C \times C$ to $\mathbb{R}$ satisfying (A1)-(A4), and let $r>0$ and $x \in E$. Then, there exists $z \in C$ such that

$$
f(z, y)+\frac{1}{r}\langle y-z, J z-J x\rangle \geq 0, \quad \forall y \in C .
$$

Lemma 2.8 (Takahashi and Zembayashi [26]). Let $C$ be a closed convex subset of a uniformly smooth, strictly convex, and reflexive Banach space $E$, and let $f$ be a bifunction from $C \times C$ to $\mathbb{R}$ satisfying (A1)-(A4). For all $r>0$ and $x \in E$, define a mapping $T_{r}: E \rightarrow C$ as follows:

$$
T_{r} x=\left\{z \in C: f(z, y)+\frac{1}{r}\langle y-z, J z-J x\rangle \geq 0, \quad \forall y \in C\right\} .
$$

Then, the following hold:

(1) $T_{r}$ is single-valued;

(2) $T_{r}$ is a firmly nonexpansive-type mapping [12], i.e., for all $x, y \in E$,

$$
\left\langle T_{r} x-T_{r} y, J T_{r} x-J T_{r} y\right\rangle \leq\left\langle T_{r} x-T_{r} y, J x-J y\right\rangle ;
$$

(3) $F\left(T_{r}\right)=E P(f)$;

(4) $E P(f)$ is closed and convex. 
Lemma 2.9 (Takahashi and Zembayashi [26]). Let $C$ be a closed convex subset of a smooth, strictly, and reflexive Banach space $E$, let $f$ be a bifucntion from $C \times C$ to $\mathbb{R}$ satisfying (A1) - (A4), let $r>0$. Then, for all $x \in E$ and $q \in F\left(T_{r}\right)$,

$$
\phi\left(q, T_{r} x\right)+\phi\left(T_{r} x, x\right) \leq \phi(q, x) .
$$

\section{Main Results}

In this section, we prove the strong convergence theorem.

Theorem 3.1. Let $E$ be a uniformly convex and uniformly smooth Banach space, let $C$ be a nonempty closed convex subset of $E$. Let $f$ be a bifunction from $C \times C$ to $\mathbb{R}$ satisfying (A1)-(A4), and let $\left\{T_{i}\right\}_{i=1}^{\infty}$ be an infinitely countable family of closed relatively quasi-nonexpansive mappings from $C$ into itself. Assume that $F:=\bigcap_{i=1}^{\infty} F\left(T_{i}\right) \cap E P(f) \neq \emptyset$. For an initial point $x_{0} \in E$ with $x_{1}=\Pi_{C_{1}} x_{0}$ and $C_{1}=C$, define a sequence $\left\{x_{n}\right\}$ as follows:

$$
\left\{\begin{array}{l}
y_{n, i}=J^{-1}\left(\alpha_{n} J x_{1}+\left(1-\alpha_{n}\right) J T_{i} x_{n}\right), \\
u_{n, i} \in C \text { such that } f\left(u_{n, i}, y\right)+\frac{1}{r_{n}}\left\langle y-u_{n, i}, J u_{n, i}-J y_{n, i}\right\rangle \geq 0, \quad \forall y \in C, \\
C_{n+1}=\left\{z \in C_{n}: \sup _{i \geq 1} \phi\left(z, u_{n, i}\right) \leq \alpha_{n} \phi\left(z, x_{1}\right)+\left(1-\alpha_{n}\right) \phi\left(z, x_{n}\right)\right\}, \\
x_{n+1}=\prod_{C_{n+1}} x_{1}, \quad \forall n \geq 1,
\end{array}\right.
$$

where $J$ is the duality mapping on $E$. Assume that $\left\{\alpha_{n}\right\}$ and $\left\{r_{n}\right\}$ are two sequences satisfying the restrictions:

(B1) $\left\{\alpha_{n}\right\} \subset[0,1]$ and $\lim _{n \rightarrow \infty} \alpha_{n}=0$;

(B2) $\left\{r_{n}\right\} \subset[a, \infty)$ for some $a>0$.

Then, the sequence $\left\{x_{n}\right\}$ converges strongly to $\Pi_{F} x_{1}$.

Proof. We split our proof into seven steps.

Step 1. Show that $C_{n}$ is closed and convex for all $n \geq 1$.

By Lemma 2.6, we know that $F\left(T_{i}\right)$ is closed and convex for all $i=1,2, \ldots$ By Lemma 2.8 (4), we also know that $E P(f)$ is closed and convex. Hence $F:=$ $\bigcap_{i=1}^{\infty} F\left(T_{i}\right) \cap E P(f)$ is a nonempty, closed and convex subset of $C$; consequently, $\Pi_{F} x_{1}$ is well-defined. Clearly, $C_{1}=C$ is closed and convex. Suppose that $C_{k}$ is closed and convex for $k \in \mathbb{N}$. For each $z \in C_{k}$ and $i=1,2, \ldots$, we observe that $\phi\left(z, u_{k, i}\right) \leq \alpha_{k} \phi\left(z, x_{1}\right)+\left(1-\alpha_{k}\right) \phi\left(z, x_{k}\right)$ is equivalent to $2 \alpha_{k}\left\langle z, J x_{1}\right\rangle+2\left(1-\alpha_{k}\right)\left\langle z, J x_{k}\right\rangle-2\left\langle z, J u_{k, i}\right\rangle \leq \alpha_{k}\left\|x_{1}\right\|^{2}+\left(1-\alpha_{k}\right)\left\|x_{k}\right\|^{2}-\left\|u_{k, i}\right\|^{2}$.

By the construction of the set $C_{k+1}$, we see that

$$
\begin{aligned}
C_{k+1} & =\left\{z \in C_{k}: \sup _{i \geq 1} \phi\left(z, u_{k, i}\right) \leq \alpha_{k} \phi\left(z, x_{1}\right)+\left(1-\alpha_{k}\right) \phi\left(z, x_{k}\right)\right\} \\
& =\bigcap_{i=1}^{\infty}\left\{z \in C_{k}: \phi\left(z, u_{k, i}\right) \leq \alpha_{k} \phi\left(z, x_{1}\right)+\left(1-\alpha_{k}\right) \phi\left(z, x_{k}\right)\right\} .
\end{aligned}
$$

Hence, $C_{k+1}$ is closed and convex. By induction, we get that $C_{n}$ is closed and convex for all $n \geq 1$. 
Step 2. Show that $F \subset C_{n}$ for all $n \geq 1$.

$F \subset C_{1}=C$ is obvious. Suppose that $F \subset C_{k}$ for $k \in \mathbb{N}$. We note that $u_{k, i}=T_{r_{k}} y_{k, i}$ for $i=1,2, \ldots$ Then, for each $u \in F$, we have

$$
\begin{aligned}
\phi\left(u, u_{k, i}\right)= & \phi\left(u, T_{r_{k}} y_{k, i}\right) \leq \phi\left(u, y_{k, i}\right) \\
= & \phi\left(u, J^{-1}\left(\alpha_{k} J x_{1}+\left(1-\alpha_{k}\right) J T_{i} x_{k}\right)\right) \\
= & \|u\|^{2}-2\left\langle u, \alpha_{k} J x_{1}+\left(1-\alpha_{k}\right) J T_{i} x_{k}\right\rangle \\
& +\left\|\alpha_{k} J x_{1}+\left(1-\alpha_{k}\right) J T_{i} x_{k}\right\|^{2} \\
\leq & \|u\|^{2}-2 \alpha_{k}\left\langle u, J x_{1}\right\rangle-2\left(1-\alpha_{k}\right)\left\langle u, J T_{i} x_{k}\right\rangle \\
& +\alpha_{k}\left\|x_{1}\right\|^{2}+\left(1-\alpha_{k}\right)\left\|T_{i} x_{k}\right\|^{2} \\
\leq & \alpha_{k} \phi\left(u, x_{1}\right)+\left(1-\alpha_{k}\right) \phi\left(u, x_{k}\right) .
\end{aligned}
$$

Hence, $F \subset C_{k+1}$. By induction, we can conclude that $F \subset C_{n}$ for all $n \geq 1$. Hence $\Pi_{C_{n+1}} x_{1}$ is well-defined.

Step 3. Show that $\lim _{n \rightarrow \infty} \phi\left(x_{n}, x_{1}\right)$ exists.

From $x_{n}=\Pi_{C_{n}} x_{1}$ and $x_{n+1}=\Pi_{C_{n+1}} x_{1} \in C_{n+1} \subset C_{n}$, we have

$$
\phi\left(x_{n}, x_{1}\right) \leq \phi\left(x_{n+1}, x_{1}\right) \quad \forall n \geq 1 .
$$

From Lemma 2.5, we have

$$
\phi\left(x_{n}, x_{1}\right)=\phi\left(\Pi_{C_{n}} x_{1}, x_{1}\right) \leq \phi\left(u, x_{1}\right)-\phi\left(u, x_{n}\right) \leq \phi\left(u, x_{1}\right) .
$$

Combining (3.2) and (3.3), we get that $\lim _{n \rightarrow \infty} \phi\left(x_{n}, x_{1}\right)$ exists.

Step 4. Show that $\left\{x_{n}\right\}$ is a Cauchy sequence in $C$.

Since $x_{m}=\Pi_{C_{m}} x_{1} \in C_{m} \subset C_{n}$ for $m>n$, by Lemma 2.5, we have

$$
\begin{aligned}
\phi\left(x_{m}, x_{n}\right)=\phi\left(x_{m}, \Pi_{C_{n}} x_{1}\right) & \leq \phi\left(x_{m}, x_{1}\right)-\phi\left(\Pi_{C_{n}} x_{1}, x_{1}\right) \\
& =\phi\left(x_{m}, x_{1}\right)-\phi\left(x_{n}, x_{1}\right) .
\end{aligned}
$$

Taking $m, n \rightarrow \infty$, we obtain that $\phi\left(x_{m}, x_{n}\right) \rightarrow 0$. From Lemma 2.3, we have $\left\|x_{m}-x_{n}\right\| \rightarrow 0$ as $m, n \rightarrow \infty$. Hence $\left\{x_{n}\right\}$ is a Cauchy sequence. By the completeness of $E$ and the closedness of $C$, we can assume that $x_{n} \rightarrow q \in C$ as $n \rightarrow \infty$. Further, we obtain

$$
\lim _{n \rightarrow \infty} \phi\left(x_{n+1}, x_{n}\right)=0 .
$$

Note that $\lim _{n \rightarrow \infty} \alpha_{n}=0$. Since $x_{n+1}=\Pi_{C_{n+1}} x_{1} \in C_{n+1}$, we have

$$
\phi\left(x_{n+1}, u_{n, i}\right) \leq \alpha_{n} \phi\left(x_{n+1}, x_{1}\right)+\left(1-\alpha_{n}\right) \phi\left(x_{n+1}, x_{n}\right) \rightarrow 0,
$$

as $n \rightarrow \infty$. Applying Lemma 2.3 to (3.4) and (3.5), we get

$$
\lim _{n \rightarrow \infty}\left\|u_{n, i}-x_{n}\right\|=0 \quad \forall i=1,2, \ldots
$$


This shows that $u_{n, i} \rightarrow q$ as $n \rightarrow \infty$ and $\mathrm{i}=1,2, \ldots$ Since $J$ is uniformly norm-tonorm continuous on bounded subsets of $E$, we also obtain

$$
\lim _{n \rightarrow \infty}\left\|J u_{n, i}-J x_{n}\right\|=0 \quad \forall i=1,2, \ldots
$$

Step 5. Show that $q \in \bigcap_{i=1}^{\infty} F\left(T_{i}\right)$.

From (3.1), we observe that

$$
\phi\left(u, y_{n, i}\right) \leq \alpha_{n} \phi\left(u, x_{1}\right)+\left(1-\alpha_{n}\right) \phi\left(u, x_{n}\right) .
$$

Note that $u_{n, i}=T_{r_{n}} y_{n, i}$. Hence, it follows from (3.8) and Lemma 2.9 that

$$
\begin{aligned}
\phi\left(u_{n, i}, y_{n, i}\right) & =\phi\left(T_{r_{n}} y_{n, i}, y_{n, i}\right) \\
& \leq \phi\left(u, y_{n, i}\right)-\phi\left(u, T_{r_{n}} y_{n, i}\right) \\
& \leq \alpha_{n} \phi\left(u, x_{1}\right)+\left(1-\alpha_{n}\right) \phi\left(u, x_{n}\right)-\phi\left(u, u_{n, i}\right) \\
& =\alpha_{n}\left(\phi\left(u, x_{1}\right)-\phi\left(u, x_{n}\right)\right)+\left(\phi\left(u, x_{n}\right)-\phi\left(u, u_{n, i}\right)\right) .
\end{aligned}
$$

From (3.6) and (B1), we get $\lim _{n \rightarrow \infty} \phi\left(u_{n, i}, y_{n, i}\right)=0$ for all $i=1,2, \ldots$ By Lemma 2.3, we also obtain

$$
\lim _{n \rightarrow \infty}\left\|u_{n, i}-y_{n, i}\right\|=0 \quad \forall i=1,2, \ldots
$$

Again, from (3.6) and (3.9), we have

$$
\lim _{n \rightarrow \infty}\left\|x_{n}-y_{n, i}\right\|=0 \quad \forall i=1,2, \ldots
$$

and hence,

$$
\lim _{n \rightarrow \infty}\left\|J x_{n}-J y_{n, i}\right\|=0 \quad \forall i=1,2, \ldots
$$

Observing

$$
\begin{aligned}
\left\|J y_{n, i}-J x_{n}\right\| & =\left\|\alpha_{n} J x_{1}+\left(1-\alpha_{n}\right) J T_{i} x_{n}-J x_{n}\right\| \\
& =\left\|-\alpha_{n}\left(J T_{i} x_{n}-J x_{1}\right)+\left(J T_{i} x_{n}-J x_{n}\right)\right\| \\
& \geq-\alpha_{n}\left\|J T_{i} x_{n}-J x_{1}\right\|+\left\|J T_{i} x_{n}-J x_{n}\right\|,
\end{aligned}
$$

we obtain, by (B1) and (3.10), that

$$
\left\|J T_{i} x_{n}-J x_{n}\right\| \leq\left\|J y_{n, i}-J x_{n}\right\|+\alpha_{n}\left\|J T_{i} x_{n}-J x_{1}\right\| \rightarrow 0,
$$

as $n \rightarrow \infty$ and $i=1,2, \ldots$ Since $J^{-1}$ is also uniformly norm-to-norm continuous on bounded sets, we have

$$
\lim _{n \rightarrow \infty}\left\|T_{i} x_{n}-x_{n}\right\|=0 \quad \forall i=1,2, \ldots
$$

Since $T_{i}$ is closed for $i=1,2, \ldots$ and $x_{n} \rightarrow q$, we can conclude that $q \in \bigcap_{i=1}^{\infty} F\left(T_{i}\right)$.

Step 6. Show that $q \in E P(f)$.

From (3.9) and $r_{n} \geq a>0$, we have $\frac{\left\|J u_{n, i}-J y_{n, i}\right\|}{r_{n}} \rightarrow 0$. From $u_{n, i}=T_{r_{n}} y_{n, i}$ for $i=1,2, \ldots$, we get

$$
f\left(u_{n, i}, y\right)+\frac{1}{r_{n}}\left\langle y-u_{n, i}, J u_{n, i}-J y_{n, i}\right\rangle \geq 0, \quad \forall y \in C .
$$


By (A2), we have

$$
\begin{aligned}
\left\|y-u_{n, i}\right\| \frac{\left\|J u_{n, i}-J y_{n, i}\right\|}{r_{n}} & \geq \frac{1}{r_{n}}\left\langle y-u_{n, i}, J u_{n, i}-J y_{n, i}\right\rangle \\
& \geq-f\left(u_{n, i}, y\right) \geq f\left(y, u_{n, i}\right), \quad \forall y \in C .
\end{aligned}
$$

From (A4) and $u_{n, i} \rightarrow q$ for $i=1,2, \ldots$, we get $f(y, q) \leq 0$ for all $y \in C$. For $0<t<1$ and $y \in C$, Define $y_{t}=t y+(1-t) q$. Then $y_{t} \in C$, which implies that $f\left(y_{t}, q\right) \leq 0$. From $(\mathrm{A} 1)$, we obtain that $0=f\left(y_{t}, y_{t}\right) \leq t f\left(y_{t}, y\right)+(1-t) f\left(y_{t}, q\right) \leq$ $t f\left(y_{t}, y\right)$. Thus, $f\left(y_{t}, y\right) \geq 0$. From (A3), we have $f(q, y) \geq 0$ for all $y \in C$. Hence $q \in E P(f)$

Step 7. Show that $q=\Pi_{F} x_{1}$.

From $x_{n}=\Pi_{C_{n}} x_{1}$, we have

$$
\left\langle J x_{1}-J x_{n}, x_{n}-z\right\rangle \geq 0 \quad \forall z \in C_{n} .
$$

Since $F \subset C_{n}$, we also have

$$
\left\langle J x_{1}-J x_{n}, x_{n}-u\right\rangle \geq 0 \quad \forall u \in F .
$$

By taking limit in (3.11), we obtain that

$$
\left\langle J x_{1}-J q, q-u\right\rangle \geq 0 \quad \forall u \in F .
$$

By Lemma 2.4, we can conclude that $q=\Pi_{F} x_{1}$. This completes the proof.

Remark 3.2. If we take $f \equiv 0$ and $T_{i}=T$ for all $i=1,2, \ldots$, then Theorem 3.1 reduces to Theorem 3.1 of Qin et al. [19].

Remark 3.3. If we take $f \equiv 0$ and $T_{i}=T$ for all $i=1,2, \ldots$, then Theorem 3.1 improves on Theorem 3.2 of Qin and $\mathrm{Su}$ [20] from the class of relatively nonexpansive mappings to the class of relatively quasi-nonexpansive mappings; that is, we relax the strong restriction: $\hat{F}(T)=F(T)$. Moreover, this algorithm is also simpler to compute than the one given in [20].

\section{Applications}

In this section, we give some applications of Theorem 3.1 in the framework of Banach spaces.

Let $A: C \rightarrow E^{*}$ be a nonlinear mapping. The classical variational inequality problem is to find $\hat{x} \in C$ such that

$$
\langle A \hat{x}, y-\hat{x}\rangle \geq 0 \quad \forall y \in C .
$$

The set of solutions of (4.1) is denoted by $V I(C, A)$.

Theorem 4.1. Let $E$ be a uniformly convex and uniformly smooth Banach space, let $C$ be a nonempty closed convex subset of $E$. Let $A$ be a continuous monotone mapping from $C$ to $E^{*}$, and let $\left\{T_{i}\right\}_{i=1}^{\infty}$ be an infinitely countable family of closed relatively quasi-nonexpansive mappings from $C$ into itself such that 
$F:=\bigcap_{i=1}^{\infty} F\left(T_{i}\right) \cap V I(C, A) \neq \emptyset$. For an initial point $x_{0} \in E$ with $x_{1}=\Pi_{C_{1}} x_{0}$ and $C_{1}=C$, define a sequence $\left\{x_{n}\right\}$ as follows:

$$
\left\{\begin{array}{l}
y_{n, i}=J^{-1}\left(\alpha_{n} J x_{1}+\left(1-\alpha_{n}\right) J T_{i} x_{n}\right), \\
u_{n, i} \in C \text { such that }\left\langle A u_{n, i}, y-u_{n, i}\right\rangle+\frac{1}{r_{n}}\left\langle y-u_{n, i}, J u_{n, i}-J y_{n, i}\right\rangle \geq 0, \quad \forall y \in C, \\
C_{n+1}=\left\{z \in C_{n}: \sup _{i \geq 1} \phi\left(z, u_{n, i}\right) \leq \alpha_{n} \phi\left(z, x_{1}\right)+\left(1-\alpha_{n}\right) \phi\left(z, x_{n}\right)\right\}, \\
x_{n+1}=\Pi_{C_{n+1}} x_{1}, \quad \forall n \geq 1,
\end{array}\right.
$$

where $J$ is the duality mapping on $E$. Assume that $\left\{\alpha_{n}\right\}$ and $\left\{r_{n}\right\}$ are sequences satisfying (B1) and (B2) of Theorem 3.1.

Then, the sequence $\left\{x_{n}\right\}$ converges strongly to $\Pi_{F} x_{1}$.

Proof. Define $f(x, y)=\langle A x, y-x\rangle$ for all $x, y \in C$. Then, by Theorem 3.1, we obtain the desired result.

Let $\varphi: C \rightarrow \mathbb{R}$ be a real-valued function. The convex minimization problem is to find $\hat{x} \in C$ such that

$$
\varphi(\hat{x}) \leq \varphi(y) \quad \forall y \in C .
$$

The set of solutions of (4.2) is denoted by $C M P(\varphi)$.

Theorem 4.2. Let $E$ be a uniformly convex and uniformly smooth Banach space, let $C$ be a nonempty closed convex subset of $E$. Let $\varphi$ be a proper lower semicontinuous and convex function from $C$ to $\mathbb{R}$, and let $\left\{T_{i}\right\}_{i=1}^{\infty}$ be an infinitely countable family of closed relatively quasi-nonexpansive mappings from $C$ into itself such that $F:=\bigcap_{i=1}^{\infty} F\left(T_{i}\right) \cap C M P(\varphi) \neq \emptyset$. For an initial point $x_{0} \in E$ with $x_{1}=\Pi_{C_{1}} x_{0}$ and $C_{1}=C$, define a sequence $\left\{x_{n}\right\}$ as follows:

$$
\left\{\begin{array}{l}
y_{n, i}=J^{-1}\left(\alpha_{n} J x_{1}+\left(1-\alpha_{n}\right) J T_{i} x_{n}\right), \\
u_{n, i} \in C \text { such that } \varphi(y)+\frac{1}{r_{n}}\left\langle y-u_{n, i}, J u_{n, i}-J y_{n, i}\right\rangle \geq \varphi\left(u_{n, i}\right), \quad \forall y \in C, \\
C_{n+1}=\left\{z \in C_{n}: \sup _{i \geq 1} \phi\left(z, u_{n, i}\right) \leq \alpha_{n} \phi\left(z, x_{1}\right)+\left(1-\alpha_{n}\right) \phi\left(z, x_{n}\right)\right\}, \\
x_{n+1}=\prod_{C_{n+1}} x_{1}, \quad \forall n \geq 1,
\end{array}\right.
$$

where $J$ is the duality mapping on $E$. Assume that $\left\{\alpha_{n}\right\}$ and $\left\{r_{n}\right\}$ are sequences satisfying (B1) and (B2) of Theorem 3.1.

Then, the sequence $\left\{x_{n}\right\}$ converges strongly to $\Pi_{F} x_{1}$.

Proof. Define $f(x, y)=\varphi(y)-\varphi(x)$ for all $x, y \in C$. Then, by Theorem 3.1, we obtain the desired result.

As a direct consequence of Theorem 3.1 , we also obtain the following application in a Hilbert space.

Theorem 4.3. Let $C$ be a nonempty and closed convex subset of a real Hilbert space $H$. Let $f$ be a bifunction from $C \times C$ to $\mathbb{R}$ satisfying (A1)-(A4) and let $\left\{T_{i}\right\}_{i=1}^{\infty}$ be an infinitely countable family of closed quasi-nonexpansive mappings 
from $C$ into itself such that $F:=\bigcap_{i=1}^{\infty} F\left(T_{i}\right) \cap E P(f) \neq \emptyset$. For an initial point $x_{0} \in H$ with $x_{1}=P_{C_{1}} x_{0}$ and $C_{1}=C$, define a sequence $\left\{x_{n}\right\}$ as follows:

$$
\left\{\begin{array}{l}
u_{n, i} \in C \text { such that } f\left(u_{n, i}, y\right)+\frac{1}{r_{n}}\left\langle y-u_{n, i}, u_{n, i}-y_{n, i}\right\rangle \geq 0, \quad \forall y \in C, \\
y_{n, i}=\alpha_{n} x_{1}+\left(1-\alpha_{n}\right) T_{i} x_{n}, \\
C_{n+1}=\left\{z \in C_{n}: \sup _{i \geq 1}\left\|z-u_{n, i}\right\|^{2} \leq \alpha_{n}\left\|z-x_{1}\right\|^{2}+\left(1-\alpha_{n}\right)\left\|z-x_{n}\right\|^{2}\right\}, \\
x_{n+1}=P_{C_{n+1}} x_{1}, \quad \forall n \geq 1,
\end{array}\right.
$$

where $P$ is a metric projection. Assume that $\left\{\alpha_{n}\right\}$ and $\left\{r_{n}\right\}$ are sequences satisfying (B1) and (B2) of Theorem 3.1.

Then, the sequence $\left\{x_{n}\right\}$ converges strongly to $P_{F} x_{1}$.

Proof. By taking $E=H$ in Theorem 3.1, we obtain the desired result.

Remark 4.4. If we take $f \equiv 0$ and $T_{i}=T$ for all $i=1,2, \ldots$ in Theorem 4.3, then Theorem 4.3 improves and extends Theorem 3.1 of Martinez-Yanes and Xu 13.

Acknowledgements: The authors would like to thank the referee for the valuable suggestion and the Thailand Research Fund for their financial support during the preparation of this paper. The first author was supported by the Royal Golden Jubilee Grant PHD/0261/2551 and by the Graduate School, Chiang Mai University, Thailand.

\section{REFERENCES}

[1] Ya.I Alber, Matric and generalized projection operators in Banach spaces: Properties and applications, in: A.G.Kartsatos(Ed.), Theory and Applications of Nonlinear Operator of Accretive and Monotone Type, Marcel Dekker, New York, 1996.

[2] Ya.I Alber, S. Reich, An iterative method for solving a class of nonlinear operator equations in Banach spaces, Panamer. Math. J. 4 (1994) 39-54.

[3] D. Butnariu, S. Reich, A.J. Zaslavski, Asymptotic behavior of relatively nonexpansive operators in Banach spaces, J. Appl. Anal. 7 (2001) 151-174.

[4] D. Butnariu, S. Reich, A.J. Zaslavski, Weak convergence of orbits of nonlinear operators in reflexive Banach spaces, Numer. Funct. Anal. Optim. 24 (2003) 489-508.

[5] E. Blum, W. Oettli, From optimization and variational inequalities to equilibrium problems, Math. Student 63 (1994) 123-145.

[6] Y. Censor, S. Reich, Iterations of paracontractions and firmly nonexpansive operators with applications to feasibility and optimization, Optimization 37 (1996) 323-339.

[7] P. Cholamjiak, A hybrid iterative scheme for equilibrium problems, variational inequality problems and fixed point problems in Banach spaces, Fixed Point Theory Appl. (2009), doi: $10.1155 / 2009 / 719360$.

[8] I. Cioranescu, Geometry of Banach spaces, Duality Mappings and Nonlinear Problems, Kluwer Academic Plublishers, Dordrecht, 1990.

[9] P.L. Combettes, S.A. Hirstoaga, Equilibrium programming in Hilbert spaces, J. Nonlinear Convex Anal. 6 (2005) 117-136.

[10] B. Halpern, Fixed points of nonexpanding maps, Bull. Amer. Math. Soc. 73 (1967) 957-961.

[11] S. Kamimura, W. Takahashi, Strong convergence of a proximal-type algorithm in a Banach space, SIAM J. Optim. 13 (2002) 938-945.

[12] F. Kohsaka, W.Takahashi, Existence and approximation of fixed points of firmly nonexpansive type mappings in Banach spaces, SIAM J. Optim. 19 (2008) 824-835. 
[13] C. Martinez-Yanes, H.K. Xu, Strong convergence of the CQ method for fixed point iteration processes, J. Nonlinear Anal. 64 (2006) 2400-2411.

[14] S. Matsushita, W. Takahashi, A strong convergence theorem for relatively nonexpansive mappings in a Banach space, J. Approx. Theory 134 (2005) 257-266.

[15] S. Matsushita, W. Takahashi, Weak and strong convergence theorems for relatively nonexpansive mappings in Banach spaces, Fixed point Theory Appl. (2004) 37-47.

[16] K. Nakajo, W. Takahashi, Strong convergence theorems for nonexpansive mappings and nonexpansive semigroups, J. Math. Anal. Appl. 279 (2003) 372-379.

[17] S. Plubtieng, K. Ungchittrakool, Strong convergence theorems for a common fixed point of two relatively nonexpansive mappings in a Banach space, J. Approx. Theory. 149 (2007) 103-115.

[18] X. Qin, Y.J. Cho, S.M. Kang, Convergence theorems of common elements for equilibrium problems and fixed point problems in Banach spaces, J. Comput. Appl. Math. 225 (2009) 20-30.

[19] X. Qin, Y.J. Cho, S.M. Kang, H. Zhou, Convergence of a modified Halpern-type iteration algorithm for quasi- $\phi$-nonexpansive mappings, Appl. Math. Lett. 22 (2009) 1051-1055.

[20] X. Qin, Y. Su, Strong convergence theorems for relatively nonexpansive mappings in a Banach space, J. Nonlinear Anal. 67 (2007) 1958-1965.

[21] S. Reich, A weak convergence theorem for the alternating method with Bregman distance, in: A.G.Kartsatos(Ed.), Theory and Applications of Nonlinear Operator of Accretive and Monotone Type, Marcel Dekker, New York, 1996, pp. 313-318.

[22] Y. Su, D. Wang, M. Shang, Strong convergence of monotone hybrid algorithm for hemi-relatively nonexpansive mappings, Fixed Point Theory Appl. (2008), doi:10.1155/2008/284613.

[23] A. Tada, W. Takahashi, Weak and strong convergence theorems for nonexpansive mappings and an equilibrium problem, J. Optim. Theory Appl. 133 (2007) 359-370.

[24] W. Takahashi, Nonlinear Functional Analysis, Yokohama Plublishers, 2000.

[25] S. Takahashi, W. Takahashi, Strong convergence theorem for a generalized equilibrium problem and a nonexpansive mappings in a Hilbert space, J. Nonlinear Anal. 69 (2008) 1025-1033.

[26] W. Takahashi, K. Zembayashi, Strong and weak convergence theorems for equilibrium problems and relatively nonexpansive mappings in Banach spaces, J. Nonlinear Anal. 70 (2009) 45-57.

[27] W. Takahashi, K. Zembayashi, Strong convergence theorem by a new hybrid method for equilibrium problems and relatively nonexpansive mappings, Fixed point Theory Appl. (2008), doi:10.1155/2008/528476.

[28] K. Wattanawitoon, P. Kumam, Strong convergence theorems by a new projection algorithm for fixed point problems and equilibrium problems of two relatively quasi-nonexpansive mappings, Nonlinear Analysis: Hybrid Systems, 3 (2009) 11-20.

[29] H.K. Xu, Another control condition in an iterative method for nonexpansive mappings, Bull. Austral. Math. Soc. 65 (2002) 109-113.

[30] Y. Yao, M.A. Noor, Y.C. Liou, On iterative methods for equilibrium problems, J. Nonlinear Anal. 70 (2009) 497-509.

[31] C. Zălinescu, On uniformly convex functions, J. Math. Anal. Appl. 95 (1983) 344-374.

1 Department of Mathematics, Faculty of Science, Chiang Mai University, Chiang Mai 50200, Thailand.

E-mail address: prasitch2008@yahoo.com

2 Department of Mathematics, Faculty of Science, Chiang Mai University, Chiang Mai 50200, Thailand.

E-mail address: scmti005@chiangmai.ac.th 